Pacific Journal of Mathematic

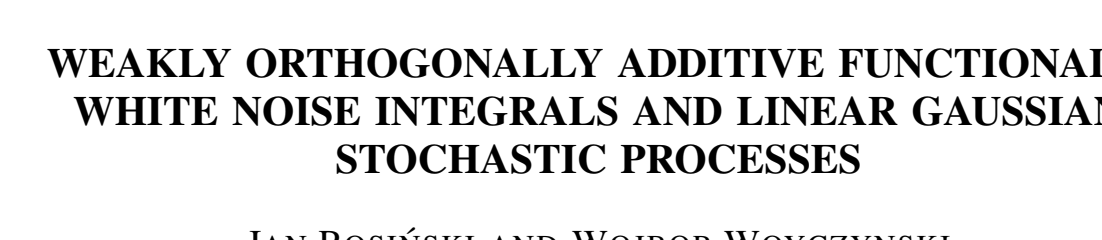




\title{
WEAKLY ORTHOGONALLY ADDITIVE FUNCTIONALS, WHITE NOISE INTEGRALS AND LINEAR GAUSSIAN STOCHASTIC PROCESSES
}

\author{
JAN ROSIŃSKI AND WOJBOR A. WOYCZYNSKI
}

\begin{abstract}
Let $E$ be a Banach space. We prove a representation theorem for functionals on $L^{2}(E)$ that are additive on weakly orthogonal elements (as defind by Beck and Warren in Pacific J. Math. in 1972) and utilize the theorem to obtain a characterization theorem for linear Gaussian stochastic processes on $L^{2}(E)$ that take independent values on weakly orthogonal functions. This enables us give a new and very natural interpretation of the notion of weak orthogonality.
\end{abstract}

1. Introduction and notation. Investigation of orthogonally additive functionals (and operators) i.e., functionals on linear spaces that are additive on orthogonal (in a certain sense) elements, has a relatively long tradition. The first paper we could find was by Pinsker [11] who in 1938 gave the representation of orthogonally additive functionals on the Hilbert space equipped with the usual pythagorean ortnogonality relation. Then there was a series of papers by Friedman, Katz, Batt, Chacon, Sundaresan, Mizel, Drewnowski, Orlicz and Woyczyński (cf. e.g., [3], [9], [12] and references quoted therein) dealing with orthogonally additive functionals and operators on various, both concrete and abstract, vector lattices where orthogonality ment disjoitness (of supports). With the same orthogonality relation, orthogonally additive functionals on the Schwartz' spaces $\mathscr{D}$ were shown by Gelfand-Vilenkin [4] to be crucial in the theory of generalized stochastic processes with independent values at each point (the latter being of utmost importance in the quantum field theory) and that direction of research was later pursued for general linear processes in [12]. At last Gudder and Strawther ([6]) introduced an axiomatic notion of orthogonality in linear spaces that, in particular, included James orthogonality. In the same paper they also gave a representation formula for orthogonally additive functionals in that situation.

We remind that they call the relation $\perp$ in a real vector space $V$ of dimension $\geqq 2$ an orthogonality if

(01) $x \perp 0,0 \perp x$ for all $x \in V$;

(02) if $x \perp y$ and $x, y \neq 0$, then $x, y$ are linearly independent;

(03) if $x \perp y$ then $a x \perp b y$ for all $a, b \in \boldsymbol{R}$;

(04) if $P$ is a two-dimensional subspace of $V$ then for every $x \in P$ there exists $0 \neq y \in P$ such that $x \perp y$; 
(05) if $P$ is a two-dimensional subspace of $V$ then there exist nonzero vectors $u, v \in P$ such that $u \perp v$ and $u+v \perp u-v$.

In the present paper we shall work with the weak orthogonality relation as introduced by Beck and Warren [2] who used it, in a subsequent paper published in Annals of Probability, to get certain strong laws of large numbers for random vectors.

Definition 1. Let $(T, \Sigma, \mu)$ be a measure space with $\mu(T)=1$, $E$ - a separable real Banach space with dual $E^{*}$, and $L^{2}(T, \Sigma, \mu ; E)=$ $L^{2}(E)$ - the Banach space of strongly $\Sigma$-measurable $E$-valued functions $f$ on $T$ for which

$$
\|f\|_{2} \stackrel{d t}{=}\left(\int_{T}\|f(t)\|^{2} \mu(d t)\right)^{1 / 2}<\infty .
$$

Two functions $f, g \in L^{2}(E)$ are said to be weakly orthogonal (denoted $f \perp^{*} g$ ) if for each $x^{*} \in E^{*}$

$$
\int_{T} x^{*} f(t) \cdot x^{*} g(t) \mu(d t)=0 .
$$

Weak orthogonality is not included in the general framework of Gudder and Strawther. The relation $\perp^{*}$ satisfies axioms (01), (02), (03) but it does not satisfy (04) and (05), except in trivial cases. That (01) and (03) are fulfilled is obvious. We prove (02). Assume, a contrario, that $f \perp^{*} g, f, g \neq 0$ and $g=a f, a \neq 0$. Let $U^{*}$ be a countable and weak* dense subset of $E^{*}$. Then for each $x^{*} \in U^{*}$

$$
\int_{T}\left[x^{*} f(t)\right]^{2} \mu(d t)=a^{-1} \int_{T} x^{*} f(t) x^{*} g(t) \mu(d t)=0 .
$$

Theorefore, for each $x^{*} \in U^{*}, x^{*} f=0 \mu$-almost everywhere so that there exists $T_{0} \subset T, \mu\left(T_{0}\right)=1$ such that for all $t \in T_{0}$ and $x^{*} \in U^{*}$, $x^{*} f(t)=0$, which in view of weak* density of $U^{*}$ in $E^{*}$ and HahnBanach theorem implies $\|f(t)\|=0$ for each $t \in T_{0}$. That means $f=0$ in $L^{2}(E)$, a contradiction. Now, we disprove (04) and (05) in the case when $\operatorname{dim} E \geqq 2$. Let $x, y \in E$ be linearly independent. Put $f=x 1_{T}, g=y 1_{T}\left(1_{A}\right.$ is the indicator function of the set $\left.A\right)$ and $P=$ $\{a f+b g: a, b \in R\} \subset L^{2}(E)$. Assume the existence of $h, 0 \neq h \in P$ such that $f \perp^{*} h, h=a f+b g$. Choose $x^{*}, y^{*} \in E^{*}$ such that $x^{*} x=y^{*} y=$ $1, x^{*} y=y^{*} x=0$. Then we have

$$
\begin{aligned}
& 0=\int_{T} x^{*} f \circ x^{*} h d \mu=a \\
& 0=\int_{T}\left(x^{*}+y^{*}\right) f \circ\left(x^{*}+y^{*}\right) h d \mu=a+b
\end{aligned}
$$

so that $h=a f+b g=0$, a contradiction. 
Thus, what we are going to prove below about weakly orthogonally additive functionals is not covered by the results of [6].

2. Representation of weakly orthogonally additive functionals.

Definition 2. We say that a function $\Phi: L^{2}(E) \rightarrow \boldsymbol{R}$ is a weakly orthogonally additive functional if for each $f, g \in L^{2}(E)$ such that $f \perp^{*} g$ we have

$$
\Phi(f+g)=\Phi(f)+\Phi(g) \text {. }
$$

We say that $\Phi$ is odd if $\Phi(-f)=-\Phi(f), f \in L^{2}(E)$, and even if $\Phi(-f)=\Phi(f)$, for all $f \in L^{2}(E)$.

In what follows we shall always assume that the measure space $T$ does not consist of a single atom i.e. that there exists $A \in \Sigma$ such that $0<\mu(A)<1$. Otherwise $f \perp^{*} g$ if and only if $f=0$ or $g=0$ so that any function $\Phi: L^{2}(E) \rightarrow R$ vanishing at 0 would be a weakly orthogonally additive functional.

THEOREM 1. If $\Phi: L^{2}(E) \rightarrow R$ is a continuous weakly orthogonally additive functional then there exists a linear, continuous, symmetric bilinear form $K(x, y)$ on $E \times E$ and an $l \in\left(L^{2}(E)\right)^{*}$ such that

$$
\Phi(f)=l(f)+\int_{T} K(f(t), f(t)) \mu(d t), \quad f \in L^{2}(E) .
$$

In the proof of the above representation theorem we shall have need of the following

LEMma 1. Let $\Phi: L^{2}(E) \rightarrow \boldsymbol{R}$ be a continuous, weakly orthogonally additive functional.

(a) If $\Phi$ is odd then it is a linear functional on $L^{2}(E)$.

(b) If $\Phi$ is even then $\Phi(a f)=a^{2} \Phi(f), a \in R, f \in L^{2}(E)$.

Proof. (a) Let $x, y \in E$ and $A \in \Sigma$ with $\mu(A)<1$. Put $a=$ $(\mu(A) / \mu(T-A))^{1 / 2}$. It is easy to check that if

$$
\begin{array}{ll}
f_{1}=x 1_{A}+a x 1_{T-A}, & f_{2}=y 1_{A}+a y 1_{T-A} \\
g_{1}=x 1_{A}+a y 1_{T-A}, & g_{2}=y 1_{A}-a x 1_{T-A}
\end{array}
$$

then $f_{1} \perp^{*} f_{2}$ and $g_{1} \perp^{*} g_{2}$ so that

$$
\begin{aligned}
& \Phi\left((x+y) 1_{A}\right)+\Phi\left(a(x-y) 1_{T-A}\right) \\
& \quad=\Phi\left(f_{1}+f_{2}\right)=\Phi\left(f_{1}\right)+\Phi\left(f_{2}\right) \\
& \quad=\Phi\left(x 1_{A}\right)+\Phi\left(a x 1_{T-A}\right)+\Phi\left(y 1_{A}\right)-\Phi\left(a y 1_{T-A}\right)
\end{aligned}
$$

and 


$$
\begin{aligned}
& \Phi\left((x+y) 1_{A}\right)-\Phi\left(a(x-y) 1_{T-A}\right) \\
& \quad=\Phi\left(g_{1}+g_{2}\right)=\Phi\left(g_{1}\right)+\Phi\left(g_{2}\right) \\
& =\Omega\left(x 1_{A}\right)+\Phi\left(a y 1_{T-A}\right)+\Phi\left(y 1_{A}\right)-\Phi\left(a x 1_{T-A}\right) .
\end{aligned}
$$

Adding up the above equalities we get that

$$
\Phi\left((x+y) 1_{A}\right)=\Phi\left(x 1_{A}\right)+\Phi\left(y 1_{A}\right) .
$$

If $\mu(A)=1$, then, by our assumption, we can find $A_{0} \in \Sigma, 0<\mu\left(A_{0}\right)<$ 1 , so that, in view of the above reasoning we also get that

$$
\begin{aligned}
\Phi\left((x+y) 1_{A}\right) & =\Phi\left((x+y) 1_{A A 0}\right)+\Phi\left((x+y) 1_{A\left(T-A_{0}\right)}\right) \\
& =\Phi\left(x 1_{A A_{0}}\right)+\Phi\left(y 1_{A A_{0}}\right)+\Phi\left(x 1_{A(T-A)}\right)+\Phi\left(y 1_{A\left(T-A_{0}\right)}\right) \\
& =\Phi\left(x 1_{A}\right)+\Phi\left(y 1_{A}\right) .
\end{aligned}
$$

Thus for any simple function in $L^{2}(E)$, and eventually, by continuity of $\Phi$, for any $f, g \in L^{2}(E)$ (simple functions $\Sigma x_{i} 1_{A i}$ are dense in $\left.L^{2}(E)\right) \Phi(f+g)=\Phi(f)+\Phi(g)$. On the other hand, by the classical theorem due to Sierpiński, every continuous additive functional on a Banach space is necessarily linear.

(b) Because of continuity of $\Phi$ and of its additivity on functions with disjoint supports it is sufficient to prove that

$$
\Phi\left(n x 1_{A}\right)=n^{2} \Phi\left(x 1_{A}\right), \quad x \in E, A \in \Sigma, \quad n \in N .
$$

As in part (a) the proof may be reduced to the case where $0<\mu(A)<1$ and we make this restriction. The above equality is evident for $n=0$ and $n=1$ and we prove it in full generality by induction. Assume, it is valid for $k, 0 \leqq k \leqq n$. Put $a=\left[\mu(A) / \mu(T-A)^{1 / 2}\right.$ and

$$
f_{n}=x 1_{A}+n a x 1_{T-A}, \quad g_{n}=n x 1_{A}-a x 1_{T-A}, \quad n=1,2, \cdots .
$$

Then, we have that $f_{n} \perp^{*} g_{n}$ and that

$$
\begin{aligned}
\Phi\left(x 1_{A}\right) & =\Phi\left(\left(f_{1}+g_{1}\right) / 2\right)=\Phi\left(f_{1} / 2\right)+\Phi\left(g_{1} / 2\right) \\
& =\Phi\left(\left(f_{1}-g_{1}\right) / 2\right)=\Phi\left(a x 1_{T-A}\right)
\end{aligned}
$$

for every $x \in E$. Utilizing the above identity and the inductive assumption we get that

$$
\begin{aligned}
\Phi((n & \left.+1) x 1_{A}\right)+(n-1)^{2} \Phi\left(x 1_{A}\right) \\
& =\Phi\left((n+1) x 1_{A}\right)+\Phi\left((n-1) a x 1_{T-A}\right)=\Phi\left(f_{n}+g_{n}\right) \\
& =\Phi\left(f_{n}\right)+\Phi\left(g_{n}\right) \\
& =\left(x 1_{A}\right)+\Phi\left(n a x 1_{T-A}\right)+\Phi\left(n x 1_{A}\right)+\Phi\left(a x 1_{T-A}\right) \\
& =2\left(1+n^{2}\right) \Phi\left(x 1_{A}\right)
\end{aligned}
$$

which was to be proved. 
Proof of the Theorem 1. The formula

$$
\Phi(f)=\frac{1}{2}(\Phi(f)-\Phi(-f))+\frac{1}{2}(\Phi(f)+\Phi(-f))
$$

gives a decomposition of the orthogonally additive functional into odd and even parts. Both are continuous and weakly orthogonally additive. Because of Lemma 1(a) it is now sufficient to show that every continuous, even and weakly orthogonally additive functional can be represented in the form

$$
\Phi(f)=\int_{T} K(f(t), f(t)) \mu(d t)
$$

for some continuous, symmetric bilinear form $K$ on $E \times E$.

In the first step we shall show that

$$
\Phi\left(x 1_{A}\right)=\Phi\left(x 1_{T}\right) \mu(A), \quad x \in E, \quad A \in \Sigma .
$$

For $A, B \in \Sigma, A \cap B=\varnothing$ we define

$$
\begin{aligned}
& f=\mu(B)^{1 / 2} x 1_{A}+\mu(A)^{1 / 2} x 1_{B}, \\
& g=\mu(B)^{1 / 2} x 1_{A}-\mu(A)^{1 / 2} x 1_{B} .
\end{aligned}
$$

Then $f \perp^{*} g$ and the application of Lemma $1(\mathrm{~b})$ yields that

$$
\begin{gathered}
4 \mu(B) \Phi\left(x 1_{A}\right)=\Phi(f+g)=\Phi(f)+\Phi(g) \\
=2 \mu(B) \Phi\left(x 1_{A}\right)+2 \mu(A) \Phi\left(x 1_{B}\right)
\end{gathered}
$$

so that

$$
\mu(B) \Phi\left(x 1_{A}\right)=\mu(A) \Phi\left(x 1_{B}\right) .
$$

In the case $B=T-A$

$$
(1-\mu(A)) \Phi\left(x 1_{A}\right)=\mu(A)\left(\Phi\left(x 1_{T}\right)-\Phi\left(x 1_{A}\right)\right)
$$

which gives (1).

In the second step, we shall check that the function $\varphi: E \rightarrow \boldsymbol{R}$ defined by the formula $\varphi(x)=\Phi\left(x 1_{T}\right)$ satisfies the functional equation

$$
\varphi(x+y)+\varphi(x-y)=2 \varphi(x)+2 \varphi(y) .
$$

Indeed, if we take $A \in \Sigma, 0<\mu(A)<1, x, y \in E$ and put

$$
a=\left[\mu(A) / \mu(T-A)^{1 / 2}, \quad f=x 1_{A}+a x 1_{T_{-A}}, \quad g=y 1_{A}-a y 1_{T_{-A}},\right.
$$

then $f \perp^{*} g$ so that, by step one and Lemma 1 (b)

$$
\begin{aligned}
& {[\varphi(x+y)+\varphi(x-y)] \mu(A)=\Phi\left((x+y) 1_{A}\right)+\Phi\left(a(x-y) 1_{T-A}\right) } \\
= & \Phi(f+g)=\Phi(f)+\Phi(g)=2 \varphi(x) \mu(A)+2 \varphi(y) \mu(A) .
\end{aligned}
$$


To conclude the proof of the Theorem 1 we shall show that

$$
K(x, y) \stackrel{d f}{=} 4^{-1}(\varphi(x+y)-\varphi(x-y)), \quad x, y \in E,
$$

is the continuonus, symmetric, bilinear form on $E \times E$ we are looking for.

The continuity of $K$ is implied by the continuity of $\varphi$ and the symmetry of $K$ by evenness of $\varphi$. Bilinearity is immediate from (2). In fact, if $x, y, z \in E$ then

$$
\begin{gathered}
4[K(x, z)+K(y, z)]=\varphi(x+z)-\varphi(x-z)+\varphi(y+z)-\varphi(y-z) \\
=2^{-1}[\varphi(x+y+2 z)-\varphi(x+y-2 z)]=2 K(x+y, 2 z),
\end{gathered}
$$

and, in particular, for $y=0$, we get that $2 K(x, z)=K(x, 2 z), x, z \in E$, from which the bilinearity is obvious. The continuity and bilinearity together imply the existence of a constant $C>0$ such that

$$
|K(x, y)| \leqq C\|x\|\|y\|, \quad x, y \in E \text {. }
$$

Now, let $f \in L^{2}(E)$ and let $f_{n}=\sum_{i} x_{i, n} 1_{A_{i, n}}$ be a sequence of simple functions tending to $f$ in $L^{2}(E)$. Then the continuity of $\varphi,(1),(4)$ and the fact that $K(x, x)=\varphi(x)$ imply that

$$
\begin{aligned}
\Phi(f) & =\lim _{n \rightarrow \infty} \Phi\left(f_{n}\right)=\lim _{n} \Phi\left(\sum_{i} x_{i, n} 1_{A_{i, n}}\right) \\
& =\lim _{n} \sum_{i} \Phi\left(x_{i, n} 1_{A_{i, n}}\right)=\lim _{n} \sum_{i} K\left(x_{i, n}, x_{i, n}\right) \mu\left(A_{i, n}\right) \\
& =\lim _{n} \int_{T} K\left(f_{n}, f_{n}\right) d \mu=\int_{T} K(f, f) d \mu
\end{aligned}
$$

which ends the proof of the Theorem 1.

REMARK 1. The method of proof employed in Theorem 1 makes it possible to prove an analogous representation theorem for weakly orthogonally additive operators taking values in another Banach space.

As far as the converse to the Theorem 1 is concerned we can prove it only in the case when $E$ has the bounded approximation property i.e. if there exists a sequence of finite dimesional operators $A_{n}: E \rightarrow E$ with uniformly bounded norms and approximating the identity operator on $E$ uniformly on compacta.

THeOREM 2. Let $E$ be a real, separable Banach space with the bounded approximation property, $K(x, y)$ a continuous, symmetric bilinear form on $E \times E$, and $l \in\left(L^{2}(E)\right)$. Then

$$
\Phi(f)=l(f)+\int_{T} K(f(t), f(t)) \mu(d t), \quad f \in L^{2}(E),
$$


is a continuous weakly orthogonally additive functional on $L^{2}(E)$.

Proof. By Pełczyński's theorem ([10]) any separable Banach space with the bounded approximation property is (isomorphic to) a complemented subspace of a Banach space with a Schauder basis. So we may assume that $E \subset F, B: F \rightarrow E$ is a bounded projection onto $E$ and that $\left(e_{i}\right)$ is a basis in $F$ with $\left(e_{\imath}^{*}\right) \subset F^{*} Q \subset E^{*}$ being a bi-orthogonal sequence. Take $f, g \in L^{2}(E)$

$$
f=B f=\sum_{i=1}^{\infty}\left(e_{i}^{*} f\right) B e_{i}, \quad g=B g=\sum_{i=1}^{\infty}\left(e_{i}^{*} g\right) B e_{i}
$$

which are weakly orthogonal. Notice that if

$$
A_{n} x=\sum_{i=1}^{n}\left(e_{i}^{*} x\right) e_{i}
$$

are the finite rank operators generated by the basis then $\left\|A_{n}\right\|$ are uniformly bounded by a constant, say $\lambda$, so that

$$
\mid K\left(B A_{n} f(t), B A_{n} g(t) \mid \leqq C\|B\|^{2} \lambda^{2}\|f(t)\|\|g(t)\| \in L^{1}(R) .\right.
$$

Therefore, by continuity of $K$, Lebesgue dominated convergence theorem, bilinearity and symmetry of $K$ (in that order) we get that

$$
\begin{aligned}
\int_{T} K(f, g) d \mu= & \lim _{n \rightarrow \infty} \int_{T} K\left(B A_{n} f, B A_{n} g\right) d \mu \\
= & \sum_{i, j=1}^{\infty} K\left(B e_{i}, B e_{j}\right) \int_{T} e_{i}^{*} f \cdot e_{j}^{*} g d \mu \\
= & \sum_{i=1}^{\infty} K\left(B e_{i}, B e_{i}\right) \int_{T} e_{i}^{*} f \cdot e_{i}^{*} g d \mu \\
& +\sum_{\substack{i, j=1 \\
i<j}}^{\infty} K\left(B e_{i}, B e_{j}\right) \int_{T}\left(e_{i}^{*} f \cdot e_{j}^{*} g+e_{j}^{*} f \cdot e_{i}^{*} g\right) d \mu \\
= & \sum_{\substack{i, j=1 \\
i<j}}^{\infty} K\left(B e_{i}, B e_{j}\right) \int_{T}\left[\left(e_{i}^{*}+e_{j}^{*}\right) f\right. \\
& \left.\cdot\left(e_{i}^{*}+e_{j}^{*}\right) g-e_{i}^{*} f \cdot e_{i}^{*} g-e_{j}^{*} f \cdot e_{j}^{*} g\right] d \mu=0
\end{aligned}
$$

which, obviously, implies the weak orthogonal additivity of $\Phi$.

REMARK 2. An additional light on the interrelations between weak orthogonality, bounded approximation property, and bilinear functionals (tensor products) is shed by the result due to Grothendieck (cf. Prop. 40 p. 180 of [5]) which characterizes the bounded approximation property of $E^{*}$ (implying the same for $E$ ) by the fact that for any Banach space $F$ the projective tensor product $E^{*} \hat{\otimes} F^{*}$ (i.e., all continuous bilinear forms on $E \times F$ ) is isomorphic to the space 
of all integral bilinear forms on $E \times F$, i.e., forms $K$ of the shape

$$
K(x, y)=\int_{B_{E^{*} \times B_{F^{*}}}} x^{*} x \cdot y^{*} y \nu_{1}\left(d x^{*}\right) \nu_{2}\left(d y^{*}\right), \quad x \in E, y \in F,
$$

where $\nu_{1}, \nu_{2}$ are certain positive measures on the unit balls $B_{E^{*}}, B_{F^{*}}$, rospectively.

REMARK 3. For any separable Banach space $E$ there exist continuous, weakly orthogonally additive functionals $\Phi$ on $L^{2}(E)$ that are strictly positive, i.e., such that $\Phi(f)>0$ for every $0 \neq f \in L^{2}(E)$. Indeed, take a sequence $\left(x_{i}\right)$ dense in $E$ and $\left(x_{i}^{*}\right) \subset E^{*}$ such that $\left\|x_{i}^{*}\right\|+1, x_{i}^{*} x_{i}=\left\|x_{i}\right\|$. Let $\nu$ be a probabilistic measure on $\left\{x^{*} \in E^{*}\right.$ : $\left.\left\|x^{*}\right\| \leqq 1\right\}$ such that $\nu\left(\left\{x_{2}^{*}\right\}\right)=2^{-i}, i=1,2, \cdots$. Define

$$
K(x, y) \stackrel{d f}{=} \int_{B_{E^{*}}} x^{*} x x^{*} y \nu\left(d x^{*}\right)
$$

Then $\Phi(f)=\int_{T} K(f, f) d \mu$ is a continuous, weakly orthogonally additive functional on $L^{2}(E)$ (cf. Th. 2) and, moreover, if $\|f\|_{2}>0$ and $t_{0} \in\{t:\|f(t)\|>0\}$ then one can find an $i$ such that $x_{i}^{*} f\left(t_{0}\right) \geqq\left\|f\left(t_{0}\right)\right\| / 2$ so that

$$
\int_{B_{E^{*}}}\left[x^{*} f\left(t_{0}\right)\right]^{2} \nu\left(d x^{*}\right) \geqq 2^{-i-1}\left\|f\left(t_{0}\right)\right\|>0,
$$

and

$$
\Phi(f)=\int_{\{t:\|f(t)\|>0\}} \int_{B_{E^{*}}}\left[x^{*} f(t)\right]^{2} \nu\left(d x^{*}\right) \mu(d t)>0 .
$$

3. A characterization of certain linear Gaussian stochastic processes. In this section we shall sketch a few probabilistic applications of the notion of weak orthogonality.

DEFINITION 3. Let $(\Omega, \mathscr{F}, P)$ be a probability space and let $L^{0}(\Omega, \mathscr{F}, P)$ be a linear space of all real random variables on $(\Omega, \mathscr{F}, P)$ equipped with the topology of convergence in probability. Any linear continuous map

$$
X: L^{2}(E) \longrightarrow L^{0}(\Omega, \mathscr{F}, P)
$$

is said to be a linear stochastic process on $L^{2}(E)$. We say that it is independently scattered (on weakly orthogonal functions) if $X(f)$ and $X(g)$ are independent random variables whenever $f \perp{ }^{*} g$. In the case when $X\left(L^{2}(E)\right) \subset L_{0}^{2}(\Omega, \mathscr{F}, P)$ (the Hilbert space of zero mean and finite variance random variables) we shall say that $X$ is orthogonally scattered if it takes orthogonal values on weakly orthogonal 
functions (cf. e.g., [1], [4], [12] for general theory of linear stochastic processes).

Notice, that if $X$ is a linear process on $L^{2}(E)$ the random function $M(x, A) \stackrel{d f}{=} X\left(x 1_{A}\right)$ on $E \times \Sigma$ has the following properties: for fixed $x_{0} \in E, M\left(x_{0}, \cdot\right)$ is contably additive, and for fixed $A_{0} \in \Sigma, M\left(\cdot, A_{0}\right)$ is linear stochastic process on $E$. So for any simple function $f=$ $\Sigma x_{i} 1_{A_{i}} \in L^{2}(E)$ we can define the linear random integral

$$
\int_{T} M(f(t), d t) \stackrel{d f}{=} \sum M\left(x_{i}, A_{i}\right)
$$

which by continuity may be extended to the whole $L^{2}(E)$. Therefore every stochastic linear process on $L^{2}(E)$ has the "generalized" random integral representation

$$
X(t)=\int_{T} M(f(t), d t) .
$$

Especially interesting examples of such linear processes are given by random integrals. This is exactly the case when the scalarly concentrated cylindrical measure on $E^{*}$ generated for each $A_{0} \in \Sigma$ by the linear process $M\left(\cdot, A_{0}\right)$ is cylindrically concentrated and can be extended to the Borel probability on $E^{*}$. Then $M$ is of the form

$$
M(x, A)=M^{*}(A) x, \quad x \in E,
$$

where $M^{*}$ is a random vector measure with values in $E^{*}$ i.e. for each $A \in \Sigma, M^{*}(A)$ is an $E^{*}$-valued random vector and the mapping $M^{*}: \Sigma \rightarrow L^{0}\left(\Omega, \mathscr{F}, P ; E^{*}\right)$ is countably additive (If it is into

$$
L_{0}^{2}\left(\Omega, \mathscr{F}, P ; E^{*}\right)
$$

we always assume that it is continuous in the $L^{2}$ norm). When the linear process $X$ is independently scattered then the corresponding random measure $M^{*}$ takes independent values on disjoint sets. If $X$ is orthogonally scattered then the random measure $M^{*}$ takes weak* orthogonal values on disjoint sets, i.e., for disjoint $A, B \in \Sigma$ and any $x, y \in E, E M^{*}(A) x M^{*}(B) y=0$. (Notice that in $L_{0}^{2}\left(\Omega, \mathscr{F}, P ; E^{*}\right)$ independence of elements implies the weak* orthogonality.) In the above case we shall also write

$$
X(t)=\int M(f(t), d t)=\int_{T} f(t) M^{*}(d t),
$$

and the latter integral (appearing only formally above) may be regarded as a Banach space analogue of the Wiener white noise integral.

What is of interest to us is that, conversely, if $M^{*}$ is any (random 
vector (measure with values in $L_{0}^{2}\left(\Omega, \mathscr{F}, P ; E^{*}\right)$ that takes weak* orthogonal (and in particular independent) values on disjoint sets then we can define the random "white noise" integral $\int f d M^{*}$ for all $f \in$ $L^{2}(T, \Sigma, \mu ; E)$, where $\mu \geqq 0$ is any measure satisfying $\left\|M^{*}(A)\right\|_{2}^{2} \leqq$ const $\mu(A), A \in \Sigma$. (The existence of such a measure is guaranteed by a theorem on the existence of a control measure for general vector measures, see e.g., Dunford and Schwartz, Linear Operators, Part I, Ch. IV. 10.) Indeed, for $f=\Sigma x_{i} 1_{A_{i}}$ put $\int f d M^{*}=\Sigma M^{*}\left(A_{i}\right) x_{i}$ and notice the inequality

$$
\begin{aligned}
E\left(\int f d M^{*}\right)^{2} & \leqq E\left(\Sigma M^{*}\left(A_{i}\right) x_{i}\right)^{2} \\
& \leqq E \Sigma\left(M^{*}\left(A_{i}\right) x_{i}\right)^{2} \leqq \Sigma E\left\|M^{*}\left(A_{i}\right)\right\|^{2}\left\|x_{i}\right\|^{2} \leqq \mathrm{const}\|f\|_{2}
\end{aligned}
$$

which permits the extension to all of $L^{2}(E)$.

We have the following neat characterization of random integrals that are orthogonally scattered linear processes.

THEOREM 3. Let the random measure $M^{*}$ takes values in $L_{0}^{2}\left(\Omega, \mathscr{F}, P ; E^{*}\right)$. Then the linear process

$$
X(f)=\int_{T} f(t) M^{*}(d t), \quad f \in L^{2}(T, \Sigma, \mu ; E)
$$

is orthogonally scattered if and only if $M^{*}$ takes weak* orthogonal values on disjoint sets and

$$
E\left(M^{*}(A) x\right)^{2}=E\left(M^{*}(T) x^{2} \cdot \mu(R) .\right.
$$

for every $x \in E$ and $A \in \Sigma$.

Proof. Assume that $X$ is orthogonally scattered. Then $\Phi(f) \stackrel{d f}{=}$ $E X^{2}(f)$ is an orthogonally additive functional and by Theorem 1 there exists $K(x, y)$ such that

$$
E X^{2}(f)=\int_{T} K(f, f) d \mu .
$$

Then

$$
E\left(M^{*}(A) x\right)^{2}=E X^{2}\left(x 1_{A}\right)=K(x, x) \mu(A)
$$

so that putting $A=T$ we get that $K(x, x)=E\left(M^{*}(T) x\right)^{2}$ which yields (5). The weak* orthogonality of $M^{*}$ is obvious because if $A \cap B=\varnothing$ then for any $x, y \in E, x 1_{A} \perp^{*} y 1_{B}$.

To prove the converse suppose $M^{*}$ is weak* orthogonal on disjoint sets and satisfies (5). Then 
$E M^{*}(A) x M^{*}(A) y=E M^{*}(T) x \cdot M^{*}(T) y \cdot \mu(A), \quad x, y \in E, A \in \Sigma$.

If $f \perp{ }^{*} g$ and $f_{n}, g_{n} \in L^{2}(E)$ are simple and such that $f_{n} \rightarrow f, g_{n} \rightarrow g$ then

$$
\begin{aligned}
E X(f) X(g) & =\lim _{n} E \int_{T} f_{n}(t) M^{*}(d t) \cdot \int_{T} g_{n}(t) M^{*}(d t) \\
& =\lim _{n} \sum_{i, j}\left[M^{*}\left(A_{i}\right) x_{i} \cdot M^{*}\left(A_{j}\right) y_{j}\right] \\
& =\lim _{n} \sum_{i} E\left(M^{*}\left(A_{\imath}\right) x_{i} \cdot M^{*}\left(A_{i}\right) y_{i}\right) \\
& =\lim _{n} \sum_{i} E M^{*}(T) x_{i} \cdot M^{*}(T) y_{i} \mu\left(A_{i}\right) \\
& =\lim _{n} E \int_{T} M^{*}(T) f_{n} \cdot M^{*}(T) g_{n} d \mu \\
& =E \int_{T} M^{*}(T) f \cdot M^{*}(T) g d \mu=0
\end{aligned}
$$

so that $X$ is orthogonally scattered and the proof is over.

Now, consider a general linear stochastic process $X$ on $L^{2}(E)$, $\mu$-atomless, which is independently scattered. Its characteristic functional

$$
\varphi(f)=E \exp (i X(f)), \quad f \in L^{2}(E),
$$

enjoys the following property

$$
f \perp^{*} g \Longrightarrow \psi(f+g)=\psi(f) \cdot \psi(g) .
$$

On the other hand, log is always exists because $X(f)$ has necessarily infinitely divisible distributions by the Central Limit Theorem (here the continuity of $X$, the fact that $\mu$ is atomless and that functions with disjoint supports are weakly orthogonal come into play). So we may define $\varphi=\log \psi$, and, obviously $\varphi$ is a complex weakly orthogonally additive functional which, by Theorem 1, must be of the form

$$
\varphi(f)=1(f)+\int K(f, f) d \mu
$$

so that the logarithm of the characteristic function of the random variable $X(f)$ is equal to

$$
\varphi(t f)=t 1(f)+t^{2} \int K(f, f) d \mu,
$$

and we have proved the following

THEOREM 4. If $X: L^{2}(T, \Sigma, \mu ; E) \rightarrow L^{0}(\Omega, \mathscr{F}, P)$, $\mu$-atomless, is a linear independently scattered stochastic process then it is necessarily 
Gaussian.

COROLLARY 1. The random integral

$$
X(f)=\int f d M^{*}, f \in L^{2}(T, \Sigma, \mu ; E),
$$

$\mu$-atomless, is independently scattered as a linear process on $L^{2}(E)$ if and only if

(i) $M^{*}$ is Gaussian;

(ii) if $A_{1}, \cdots, A_{n} \in \Sigma$ are pairwise disjoint then $M^{*}\left(A_{1}\right), \cdots$, $M^{*}\left(A_{n}\right)$ are stochastically independent;

(iii) for any $A \in \Sigma$, the random vectors $M^{*}(A)$ and $(\mu(A))^{1 / 2} M^{*}(T)$ are identically distributed.

Proof. (i) is a special case of Theorem 4 so that $M^{*}$ is necessarily Gaussian and in this case the formula (5) determines its distribution so that we get (ii) and (iii). Conversely, if $M^{*}$ is Gaussian then $E\left\|M^{*}(T)\right\|^{2}<\infty$ and by Theorem $3 X$ is orthogonally scattered and, as a Gaussian process also independently scattered.

REMARK 4. In the special case $T=[0,1]$ and $\mu$ is Lebesgue measure the above Corollary provides a characterization of the (random integral with respect to) $E^{*}$-valued Brownian motion as defined by Kuelbs in [8]. Such random integrals give us a variety of examples of independently scattered linear processes in which the notion of weak orthogonality appears very naturally.

We conclude our, somewhat sketchy, discussion of probabilistic applications of the notion of weak orthogonality by showing another instance wherein it naturally arises.

Let $E$ be a real, separable Banach space of type 2, i.e., such that for any independent, zero mean $Y_{1}, \cdots, Y_{n} \in L^{2}(\Omega, \mathscr{F}, P ; E)$

$$
E\left\|\sum_{i=1}^{n} Y_{i}\right\|_{i}^{2} \leqq C \sum_{i=1}^{n} E\left\|Y_{i}\right\|^{2}
$$

where $C$ is a universal constant, independent also of $n$ (cf. [7] for more on spaces of type 2). Assume $(T, \Sigma, \mu)$ is a measure space with $\mu(T)=1$ and $M: \Sigma \rightarrow L^{\circ}(\Omega, \mathscr{F}, P)$ is a random measure such that for any $A \in \Sigma, M(A)$ is a zero-mean Gaussian real random variable with variance $\mu(A)$ and for disjoint $A_{1}, \cdots, A_{k} \in \Sigma, M\left(A_{1}\right), \cdots, M\left(A_{k}\right)$ are independent.

Define the linear continuous mapping

$$
I: L^{2}(T, \Sigma, \mu ; E) \ni f \longrightarrow \int_{T} f(t) M(d t) \in L_{0}^{2}(\Omega, \mathscr{F}, P ; E)
$$


so that

$$
\int\left(\Sigma x_{i} 1_{A_{i}}\right) d M=\Sigma x_{i} M\left(A_{i}\right)
$$

That is possible because $E$ is of type 2 which implies that for simple functions in $L^{2}(E)$ one has the inequality

$$
E\left\|\int_{T} f d M\right\|^{2} \leqq C \int_{T}\|f\|^{2} d \mu
$$

for some $C>0$.

We shall check that the values of the random "white noise" integral (6) are independent only if integrated functions are weakly orthogonal. In fact, because $M$ is Gaussian it is implied by the fact that for each $x^{*} \in E^{*}$

$$
\begin{aligned}
E x^{*} \int_{T} f d M \cdot x^{*} \int_{T} g d M & =E \int_{T}\left(x^{*} f\right) d M \cdot \int_{T}\left(x^{*} g\right) d M \\
& =\int x^{*} f \cdot x^{*} g d \mu
\end{aligned}
$$

the penultimate equality being motivated by the fact that the mapping $L^{2}(T, \Sigma, \mu ; R) \ni f \rightarrow \int f d M \in L^{2}(\Omega, \mathscr{F}, P)$ is an isometry.

The above reasoning shows, in particular, that the integral $I$ is a linear, isometric isomorphism between $L^{2}(T, \Sigma, \mu ; E)$ and $\left.I\left[L^{2}, \Sigma, \mu ; E\right)\right] \subset$ $L_{0}^{2}(\Omega, \mathscr{F}, P ; E)$ which preserves also weak orthogonality.

\section{REFERENCES}

1. A. Badrikian, Séminaire sur les fonctions aléatoires linéaires et les measures cylindriques, Lecture Notes in Math. 139, Springer-Verlag, Berlin-Heidelberg-New York (1970).

2. A. Beck and P. Warren, Weak orthogonality, Pacific J. Math., 41 (1972), 1-11.

3. L. Drewnowski and W. Orlicz, Continuity and representation of orthogonally additive functionals, Bull. Acad. Pol. Sci., 17 (1969), 647-653.

4. I. M. Gelfand and N. Ya Vilenkin, Generalized Functions, vol. 4 Moscow (1961), Eng. transl., Academic Press, N. Y., (1964).

5. A. Grothendieck, Produits tensoriels topologiques et espaces nucléaires, Mem. Amer. Math. Soc., 16, Providence, R. I., (1955).

6. S. Gudder and D. Strawther, Orthogonally additive and orthogonally increasing functions on vector spaces, Pacific J. Math., 58 (1975), 427-436.

7. J. Hoffman-Jørgensen, Sums of independent Banach sppace valued random variables, Studia Math., 52 (1974), 159-186.

8. J. Kuelbs, Sample path behavior for Brownian notion in Banach spaces, Ann. Prob., 3 (1975), 247-261.

9. V. J. Mizel and K. Sundaresan, Representation of vector valued non-linear functions, Trans. Amer. Math. Soc., 159 (1971), 111-127.

10. A Pelczyński, Any separable Banach space with the bounded approximation property is a complemented subspace of a Banach space with a basis, Studia Math., 40 (1971), 239-242. 
11. A. G. Pinsker, Sur une fonctionelle dans l'espace de Hilbert, Comptes Redus Acad. Sci. URSS, 20 (1938), 411-414.

12. W. A. Woyczynski, Representation of additive functionals and invariant characteristic functionals of linear processes, Bull, Acad. Polon. Sci., 19 (1971), 221-230.

Received May 12, 1976 and in revised form October 26, 1976. The research of the second named author was partially supported by NSF grant \#MCS76-06935.

\section{WROC¿AW UNIVERSITY}

AND

InSTITUTE OF MATHEMATICS,

Polish ACAdemy of Sciences

Current address: W. A. Woyczynski

Northwestern University

Evanston, IL 60201 


\section{PACIFIC JOURNAL OF MATHEMATICS}

\section{EDITORS}

RICHARD ARENS (Managing Editor)

University of California

Los Angeles, California 90024

C. W. CURTIS

University of Oregon

Eugene, OR 97403

C. C. MOORE

University of California

Berkeley, CA 94720

\section{J. DugundJI}

Department of Mathematics University of Southern Californıa Los Angeles, California 90007

R. FinN AND J. Milgram Stanford University Stanford, California 94305

\section{ASSOCIATE EDITORS}

E. F. BECKENBACH

B. H. NEUMANN

F. WOLF

K. YOSHIDA

\section{SUPPORTING INSTITUTIONS}

UNIVERSITY OF BRITISH COLUMBIA CALIFORNIA INSTITUTE OF TECHNOLOGY UNIVERSITY OF CALIFORNIA MONTANA STATE UNIVERSITY UNIVERSITY OF NEVADA, RENO NEW MEXICO STATE UNIVERSITY OREGON STATE UNIVERSITY UNIVERSITY OF OREGON OSAKA UNIVERSITY
UNIVERSITY OF SOUTHERN CALIFORNIA STANFORD UNIVERSITY UNIVERSITY OF TOKYO UNIVERSITY OF UTAH WASHINGTON STATE UNIVERSITY UNIVERSITY OF WASHINGTON AMERICAN MATHEMATICAL SOCIETY NAVAL WEAPONS CENTER 


\section{Pacific Journal of Mathematics}

\section{Vol. 71, No. $1 \quad$ November, 1977}

Charalambos D. Aliprantis and Owen Sidney Burkinshaw, On universally complete Riesz spaces ............................. 1

Stephen Richard Bernfeld and Jagdish Chandra, Minimal and maximal solutions of nonlinear boundary value problems .................

John H. E. Cohn, The length of the period of the simple continued fraction of

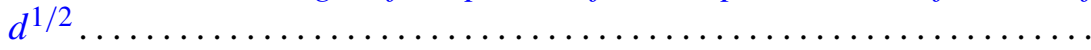

Earl Vern Dudley, Sidon sets associated with a closed subset of a compact abelian group .................................... 33

Larry Finkelstein, Finite groups with a standard component of type $J_{4} \ldots \ldots$

Louise Hay, Alfred Berry Manaster and Joseph Goeffrey Rosenstein, Concerning partial recursive similarity transformations of linearly ordered sets .......................................

Richard Michael Kane, On loop spaces without $p$ torsion. II ............

William A. Kirk and Rainald Schoneberg, Some results on

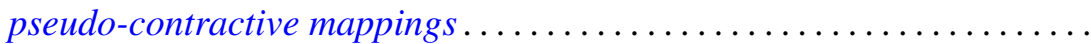

Philip A. Leonard and Kenneth S. Williams, The quadratic and quartic character of certain quadratic units. I. .

Lawrence Carlton Moore, A comparison of the relative uniform topology and the norm topology in a normed Riesz space .................

Mario Petrich, Maximal submonoids of the translational hull 119

Mark Bernard Ramras, Constructing new R-sequences . . .

Dave Riffelmacher, Multiplication alteration and related rigidity properties

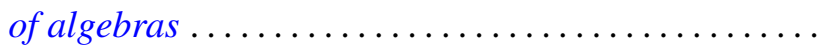

Jan Rosiński and Wojbor Woyczynski, Weakly orthogonally additive functionals, white noise integrals and linear Gaussian stochastic processes.

Ryōtarō Satō, Invariant measures for ergodic semigroups of operators

Peter John Slater and William Yslas Vélez, Permutations of the positive integers with restrictions on the sequence of differences...

Edith Twining Stevenson, Integral representations of algebraic cohomology classes on hypersurfaces ........................

Laif Swanson, Generators of factors of Bernoulli shifts . 